\title{
Design and Implementation of Video System Based on STM32
}

\author{
Yu Lin ${ }^{1,}$, Z Zhiwei Lin ${ }^{2}$ and Jing Tian $^{3}$ \\ ${ }^{1}$ The Department of Computer \& EEE Oxbridge College, Kunming University of Science and \\ Technology, Kunming, China \\ ${ }^{2}$ Faculty of Civil Engineering and Mechanic, Kunming University of Science and Technology, \\ Kunming, China \\ ${ }^{3}$ Yunnan computer software technology development research center, Kunming, China \\ akmly2011@163.com
}

Keywords: STM32; $\mu$ C/OS-II; OV7670; SCCB; FIFO

\begin{abstract}
Video acquisition system is widely used in industrial control, intelligent buildings, medical, public safety and other fields, and video communication is mainstream news of $4 \mathrm{G}$ era, for video acquisition and display technology has high social value and economic value; most of the embedded video acquisition system is based on DSP and arm technology, these systems is more complex, high cost and is not conducive to large-scale application. In this paper, the system uses the STM32 and 0v7670 camera to design of video acquisition system, analyzing the composition of the hardware system, the unique idea of the software of the system is put forward, and the stable operation of the system is realized.
\end{abstract}

\section{Introduction}

With the development of electronic products to the direction of low power consumption, low price, intelligent, based on communication technology and computer technology of real-time video acquisition has become a hot research topic. The paper according to the demand of video acquisition, video storage designs embedded video acquisition system, using the STM32 microcontroller for video information acquisition, display and storage, through the UC / OS-II embedded real-time system for task scheduling, better the management and operation of complicated procedures, so the video acquisition system has good real-time performance, low power consumption, low cost, high quality [1][2].

\section{System Design}

This system is composed of two parts video monitoring terminal and video display terminal, including video information collection, display and storage system, the work is shown in Fig. 1.

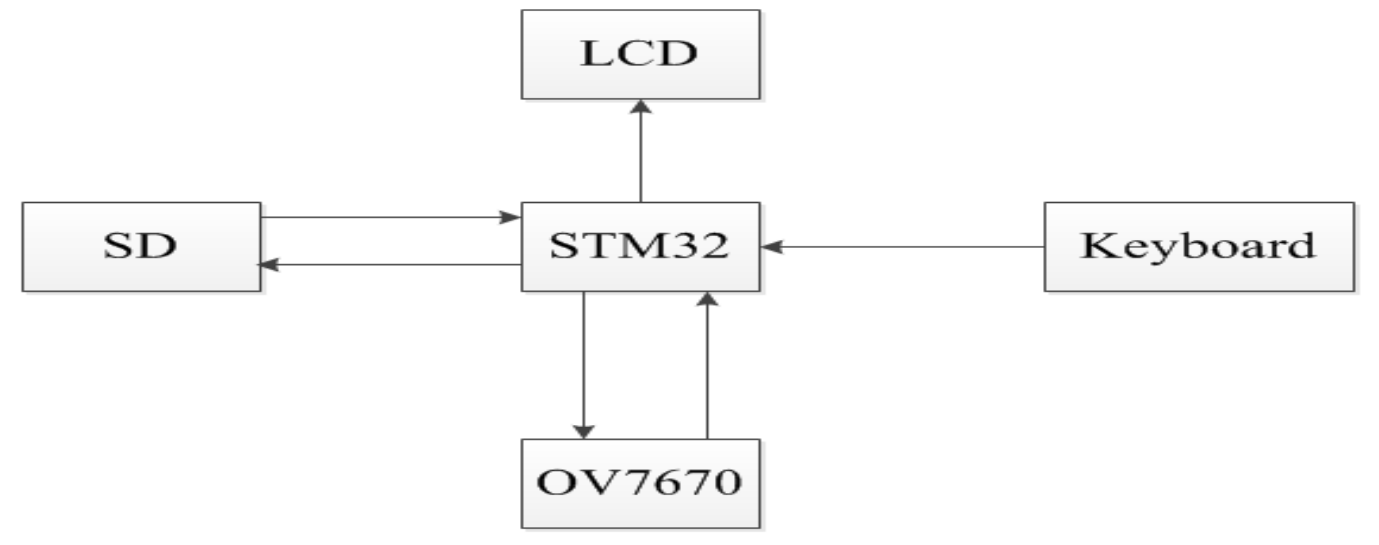

Figure 1. System working diagram 


\section{Hardware Design}

Video acquisition system is mainly composed of four modules: video acquisition, video processing, video display and storage.

mage acquisition module is mainly composed of OV7670, the video sensor has the advantages of small volume, operating voltage low, through the SCCB bus can realize gamma curve, white balance, saturation, color and video processing function, so it could output different resolution video data, by reducing or eliminating optical or electronic defects such as fixed pattern noise, Tony, and improve the video quality [3][4].

The video processing module is mainly composed of STM32F103ZET6 microcontroller which runs uC/OS-II system, and its main frequency is $72 \mathrm{MHZ}$. The video quality, data format and transmission mode can be set by configuring the OV7670 registers [5]. Through the underlying driver to make OV7670 camera acquire the video data, stored in the SD card and displayed on the LCD screen. The video display module is mainly composed of LCD screen, and the video storage module is made up of SD card [6].

\section{Software Design}

uC/OS-II is widely used in microprocessors, microcontrollers and digital signal processors. CPU hardware related parts are written in assembly language, the total amount OF assembly language is about 200 lines, in order to facilitate the transplant to any other kind of CPU. UC/OS-II is just a real-time operating system kernel, it contains the basic functions such as task scheduling, task management, time management, memory management and inter task communication and synchronization. No additional services are provided, such as input, output management, file system, network, uC/OS-II has the characteristics of high efficiency, small footprint, good real-time performance and scalability. The smallest kernel can be compiled to $2 \mathrm{~KB}$. uC/OS-II goal is to achieve a scheduling priority preemptive real-time kernel, providing the most basic system services, such as semaphores, mailbox, message queues, memory management, interrupt management. The operating system can arrange the multiple tasks to be executed in a reasonable way, and it is more efficient in multi tasks, and the uC/OS-II operating system is used to realize the task management and scheduling.

Ov7670 clock up to $24 \mathrm{MHz}$, if the system directly uses STM32 collected video information, it is very CPU intensive. So in order to reduce the load of CPU, this paper is not directly from the ov7670 read date, video data first is stored in the FIFO memory. When the camera completing a pair of videos, waiting to be processed, STM32 reads the video date from the FIFO buffer, then displays on the LCD and storage on the SD card, completion of a video data collection[7][8]. Control timing of OV7670 is Fig. 2.

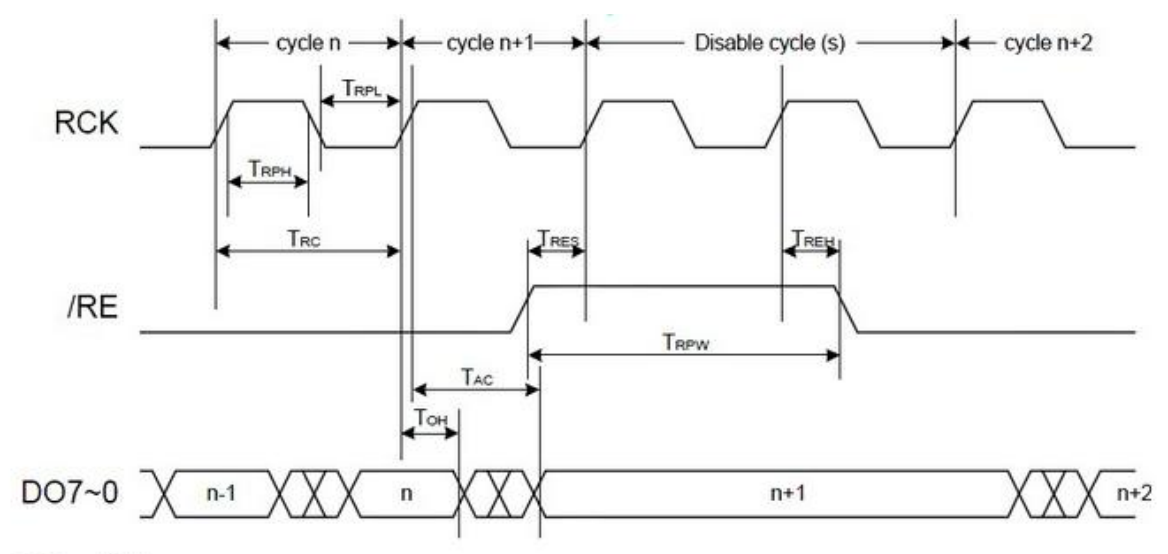

IOE $=$ "L"

Figure 2. Control timing of OV7670

STM32 through the SCCB bus achieves initial configuration to control OV7670, including 
reading and writing registers and send commands to register, waiting for the signal synchronization, is used to control the ov7670 working mode and parameters. At last it can set the camera working mode through the keyboard [9][10], and the program flow chart is shown in Fig. 3.

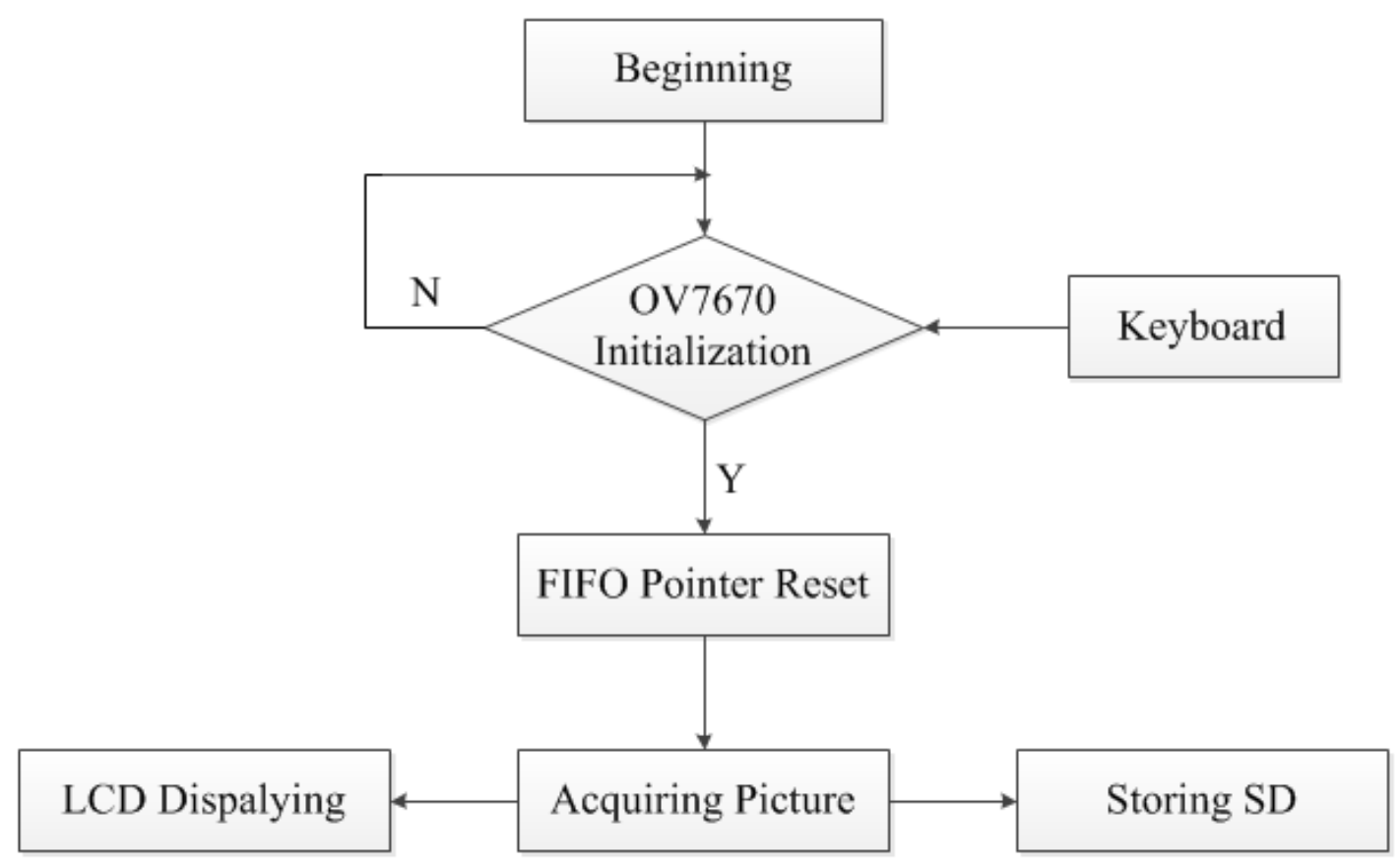

Figure 3. Program flow chart

Due to limited length, we will list some important procedures.

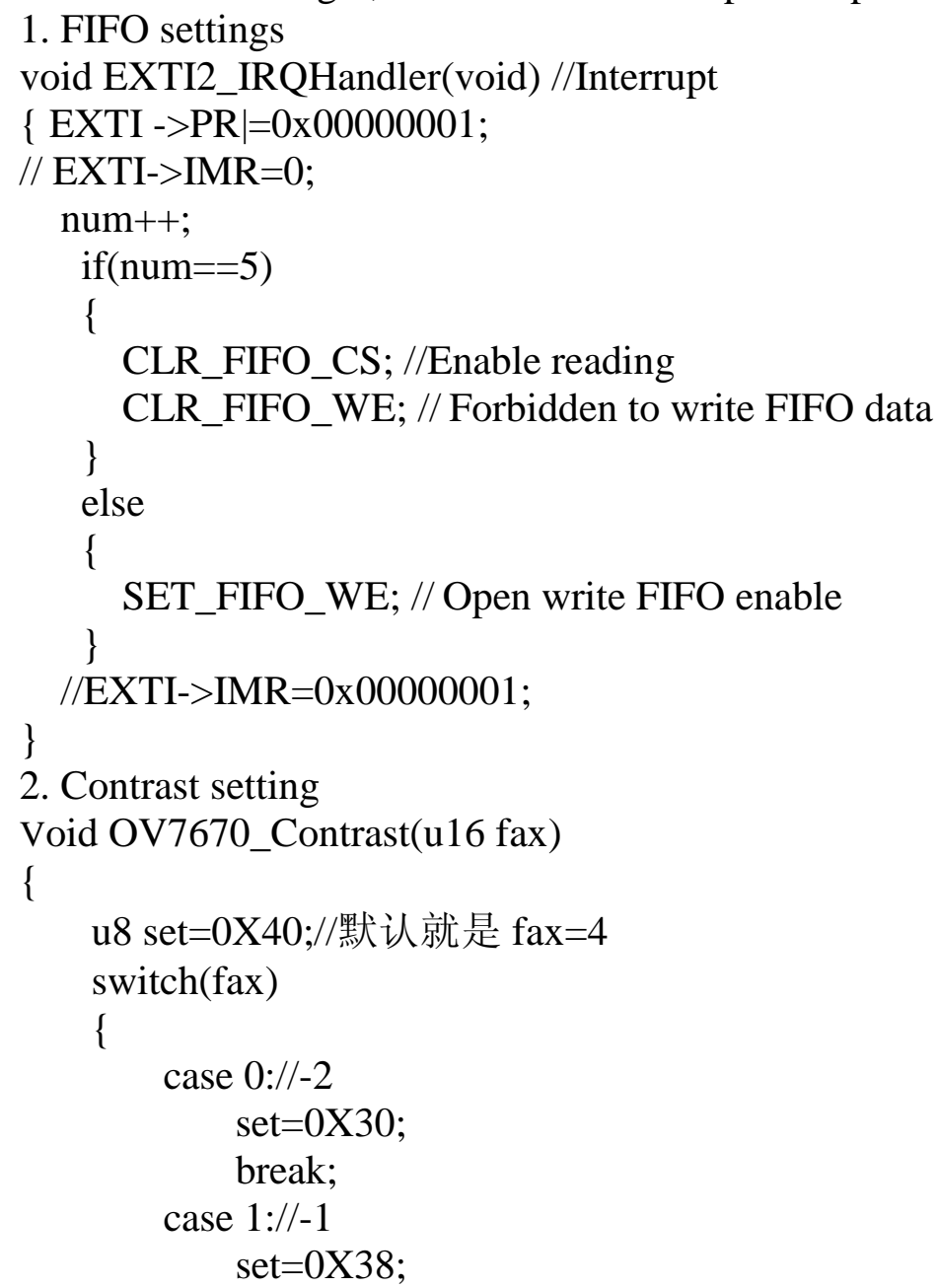




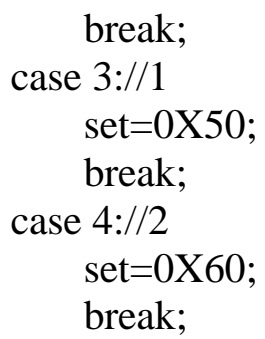

break;

case $3: / / 1$

set $=0 \times 50$;

break;

case $4: / / 2$

set $=0 \times 60$;

break;

\section{System Test}

This system first debugs to the OV7670 driver, it is mainly the register settings, display brightness, quality, mode, exposure and so on. The OV7670 and STM32 development board is connected, the following test is field synchronization signal and the line synchronization signal, and finally completed the video acquisition, the acquisition of video is shown in Fig. 4, and video quality is clear, to meet the design requirements.

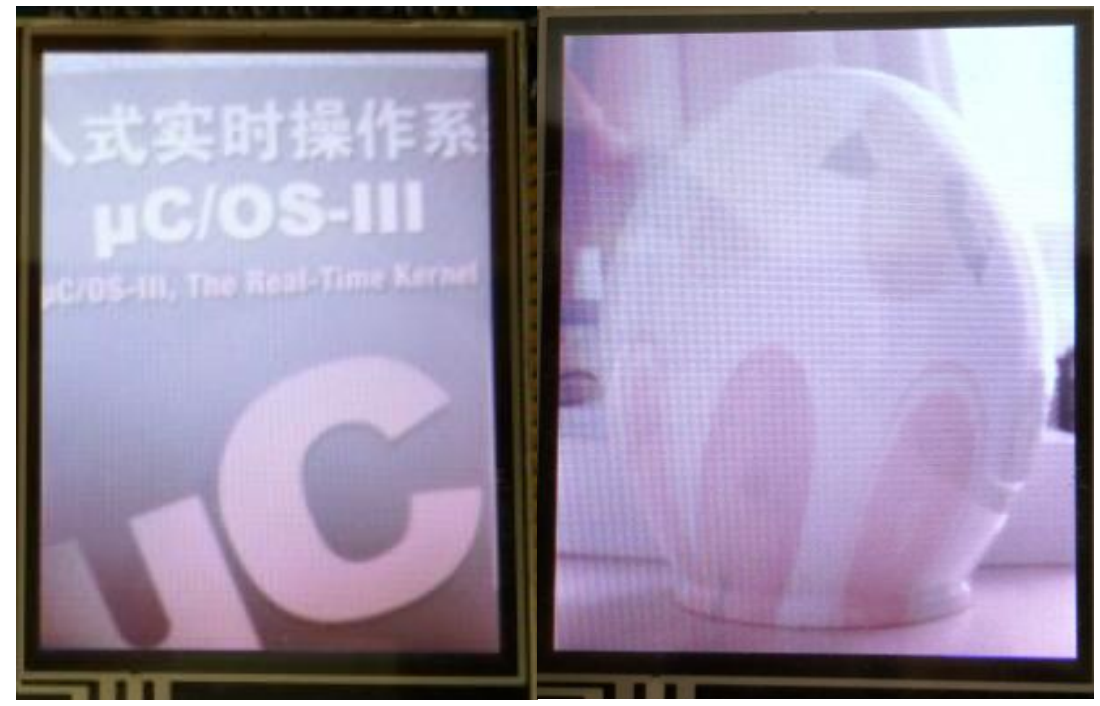

Figure 4. Video data

\section{Conclusions}

Through the above tests, the video is clear, to meet the design performance index. The advantage of this system is that hardware of the system is cost-effective, general purpose, and it has practical significance in engineering practice.

\section{References}

[1] Yunxia Gao, Yanzhang Qiu. Design of fire pump control system based on ATC89C51 [J]. Fire Science and Technology, 34(2015): 916-918.

[2] Jie Li. The Design of Wireless Video Surveillance Intelligent Car Based On STM32 [D]. Lanzhou University of Technology, 2014, 13-35.

[3] Zhong Liu. Design and Implementation of Remote Monitoring System Based on STM32 and Qt [D]. Xidian University, 2014, 17-32.

[4] Haobo Zhou. The Design of Trigger Video Acquisition and Storage System Based on STM32 [D]. Inner Mongolia University, 2014, 8-15.

[5] Yanwei Yang. Short Distance Wireless Video Communication Network Based on STM32 [J]. 
Computer Programming Skills \& Maintenance, 5(2016):31-35.

[6] Bo Mi. Research on video stream updating method based on STM32 [J]. Journal of Xi'an Aeronautical University, 32(2014):64-66.

[7] Haijiao Guo. Design of Wireless Video Monitoring System Based on ARM [D]. Xi'an Polytechnic University, 2011, 10-22.

[8] Junjie Guo, Hongying Zhu. Remote Video Control Car Based on STM32 [J]. Physical Experiment of College [J]. 4 (2015): :47-40.

[9] Bin Li. Research on Vehicle Anti Collision System Based on Video Image Processing [D]. Wuhan University of Technology, 2014, 15-30.

[10]Fushou Tao. Design and implementation of Wireless Video Surveillance Robot Based on STM32 [D]. Yunnan University, 2014, 28-40. 